\title{
Recessive multiple epiphyseal dysplasia (rMED): phenotype delineation in eighteen homozygotes for DTDST mutation R279W
}

\author{
D Ballhausen, L Bonafé, P Terhal, S L Unger, G Bellus, M Classen, B C Hamel, \\ J Spranger, B Zabel, D H Cohn, W G Cole, J T Hecht, A Superti-Furga
}

J Med Genet 2003:40:65-71

M ultiple epiphyseal dysplasia (MED) is a generalised skeletal dysplasia that although relatively mild is associated with significant morbidity. Joint pain, joint deformity, waddling gait, and short stature are the main clinical signs and symptoms. In the past, the disorder was subdivided into the milder Ribbing type, usually with flattened epiphyses, ${ }^{1}$ and the more severe Fairbank type with round epiphyses, ${ }^{2}$ but many cases were not classifiable as clearly either type. ${ }^{3}$ MED can be caused by mutations in at least six separate genes: COMP, ${ }^{4-7}$ collagen IX (COL9A1, COL9A2, and COL9A3), ${ }^{8-13}$ matrilin 3 (MATN3), ${ }^{15}$ and the sulphate transporter, DTDST (DTDST/SLC26A2). We have previously reported an adult with a recessively inherited form of MED (rMED) characterised by club feet, double layered patellae, and normal stature, who was homozygous for the mutation $862 \mathrm{c}>\mathrm{t} /$ $\mathrm{R} 279 \mathrm{~W}$ in the DTDST gene, previously associated with the achondrogenesis 1B-atelosteogenesis 2-diastrophic dysplasia spectrum. We now report on a group of 18 subjects who are homozygous for this point mutation, allowing a comprehensive assessment of this particular MED phenotype. Distinction of rMED is important because of its recessive inheritance (unlike other MED types) and genetic counselling implications. The frequency of the $\mathrm{R} 279 \mathrm{~W}$ mutation and the number of subjects with molecularly proven rMED identified since its description suggest that rMED may be more common than hitherto assumed.

\section{MATERIAL AND METHODS}

Blood or genomic DNA was sent to the Zurich centre for DTDST mutation analysis because of clinical and radiographic signs similar to the reported case of $\mathrm{rMED}^{20}$ or because of a clinical diagnosis of MED and negative mutation analysis of COMP or collagen IX genes. Genomic DNA was extracted from blood leucocytes using standard protocols and was subjected to DTDST mutation analysis. The fragment of interest, that is, the $5^{\prime}$ part of exon 3, was amplified according to the procedure previously described..$^{18}{ }^{20}$ The PCR fragment was digested with the restriction endonuclease StyI. Results were visualised on agarose gels in the presence of positive and negative controls. As the nucleotide change $862 \mathrm{c}>\mathrm{t}$ (leading to amino acid substitution R279W) creates a new restriction site for StyI, heterozygous and homozygous patients can be distinguished from wild type homozygous subjects. In all subjects with a positive StyI digestion, the genotype was confirmed by direct sequencing of the fragment amplified in a second PCR. The ABI Prism Big Dye Terminator Ready Reaction Kit and protocol were used for sequencing in an ABI Prism 310 Genetic Analyzer. In all subjects who were not homozygous for R279W, a complete DTDST mutation screening was performed ${ }^{19}$ to test for the presence of other mutations.

When R279W homozygosity was identified, the referring physician was asked to participate in this study by sending

\section{Key points}

- DTDST mutations have been identified in 28 subjects with recessive MED (rMED). Twenty-one were homozygous for R279W and seven had other genotypes. From the 21 R279W homozygotes, 18 were included in a retrospective data collection study including a questionnaire to the referring physician and radiograph review.

- Abnormal clinical findings were present at birth in only $8 / 18$ subjects. The diagnosis of a skeletal dysplasia was made in late childhood or thereafter in the majority.

- Symptoms leading to diagnosis were chronic joint pain, mild deformity of the hands and feet, scoliosis, or joint contractures.

- The height of affected subjects ranged from slightly below the 3rd centile to the 90th centile for age throughout the age range. Short stature was not a frequent finding

- The main radiographic findings were flat proximal femoral epiphyses, mild brachydactyly, and double layered patellae on lateral knee films.

- The frequency of the R279W mutation and the number of subjects with molecularly proven rMED identified since its description suggest that it may be more common than hitherto assumed.

photographs and radiographs, as well as by completing a questionnaire about family and personal history, symptoms leading to diagnosis, clinical course, present complaints, and anthropometric data. Informed consent for use of personal clinical and radiographic data in this study was obtained from all subjects in compliance with local regulations of clinical centres. When possible, parents and sibs were also genotyped in order to confirm recessive inheritance.

\section{RESULTS}

A total of 21 patients from 15 families were found to be homozygous for the R279W mutation, including three sibs reported in 1995 by Deere $\mathrm{et}^{\mathrm{l}^{23}}$ as recessive MED unlinked to COMP, as well as the original case reported,$^{20}$ For two subjects, the complete clinical information requested was not available; a third subject did not give permission to include his data in this study. Seven additional patients with clinical and radiographic signs compatible with rMED were found to carry two DTDST mutations but were not homozygous for R279W: three were homozygous for mutation $\mathrm{C} 653 \mathrm{~S}$ and one each was observed with the genotypes G237V/R279W, N77H/R279W, 


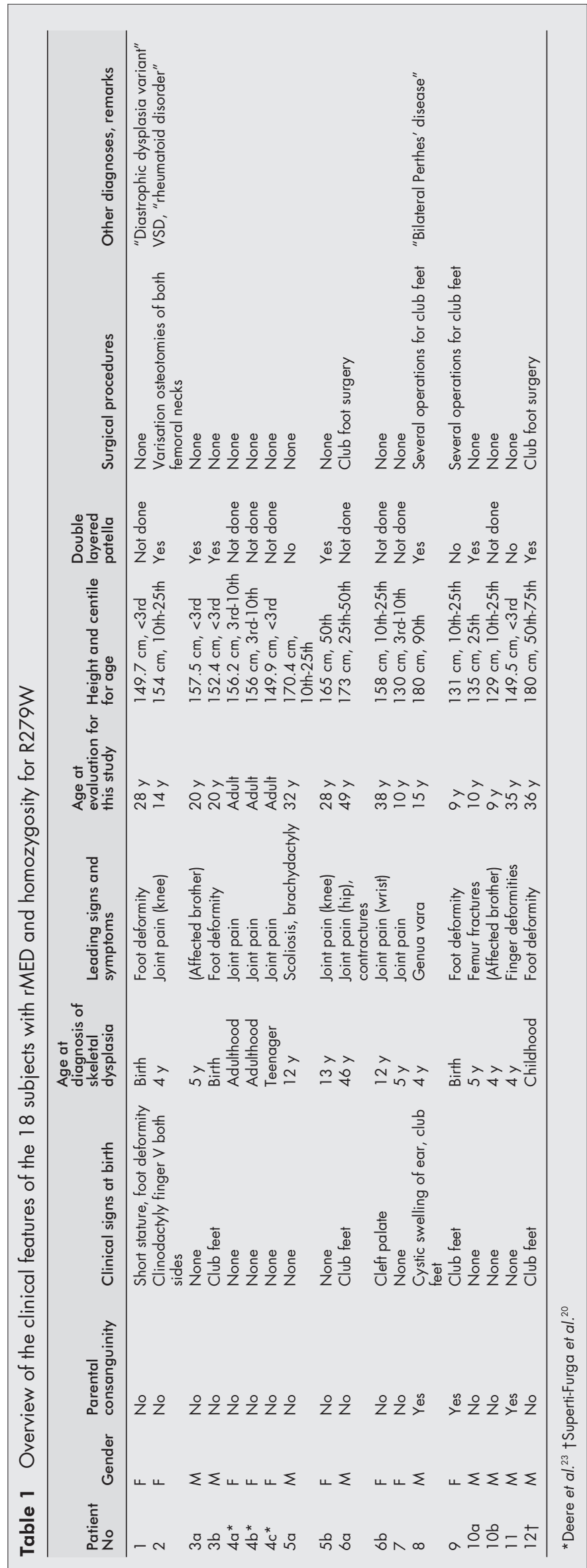

R279W/"Finnish", and "Finnish"/C635S. The phenotype of these patients was similar in all respects to that of the R279W homozygote group (including a double layered patella in all three patients in whom lateral knee films were available), but we preferred to investigate a homogeneous group in order to estimate phenotypic variability given the shared DTDST mutation, and we did not include them in our detailed analysis. Thus, the final study sample consisted of 18 R279W homozygotes from 12 families.

\section{Gender and ethnic distribution}

Of the eighteen R279W homozygotes, nine were female and nine male (table 1). The age at evaluation varied from 9 to 49 years. All patients were of Europid origin, but there were no recognisable ethnic or geographical associations. This is in accordance with the lack of ethnic association of R279W in our earlier studies. ${ }^{19}$ There was consanguinity in three of the 12 families.

\section{Clinical findings at birth}

Abnormal findings at or shortly after birth were reported in eight out of 18 cases: $4 / 18$ club foot, $1 / 18$ cleft palate, $1 / 18$ cystic ear swelling, $1 / 18$ clinodactyly. None showed the "hitch-hiker" thumbs typical of diastrophic dysplasia. The diagnosis of a "skeletal dysplasia", without further specification, had been made at birth in three out of 18 cases.

\section{Clinical course}

The majority of patients were diagnosed with a skeletal disorder in childhood. Reasons for seeking medical assistance were joint pain (8/18 cases), hand/ foot deformities (6/18), affected sibs (2/18), scoliosis $(1 / 18)$, genua vara $(1 / 18)$, and fractures $(1 / 18)$. The most frequently reported complaint was chronic joint pain in the hips, knees, wrists, and fingers. Limitation in joint movements was present in some subjects. A rheumatoid disorder was suspected in one case because of the combination of joint pain and fusiform finger swelling (fig 1C). Only one patient (patient 1 in table 1 and fig 2) had received a diagnosis of diastrophic dysplasia variant; this girl was one of the few patients whose stature was below the 3rd centile for age.

\section{Surgical interventions}

Patient 2 (table 1) had varisation osteotomies of both femoral necks performed at 8 years. Patient $6 a$ had club foot surgery at ages 6,8 , and 13 years. Patient 8 had seven operations for club foot correction. Patient 9 had club foot surgery at ages 1, 2, 8, 9, and 13 years. None of the patients has undergone hip replacement so far.

\section{Height}

Fig 2 shows the height of individual patients plotted as age related centiles. Reduced stature (below -2 SD for age) was present in five out of 18 patients. There was no association between age and the height centile score, suggesting that in most cases there was no downward crossing of the growth curve during development. Median age related height was on the 10th centile, but two subjects were of above average height.

\section{Radiographic findings} Hands (fig 3)

In contrast to other types of MED, skeletal maturation (bone age) is not delayed. There is an apparent dissociation between phalangeal epiphyses and the carpal 


\section{A}

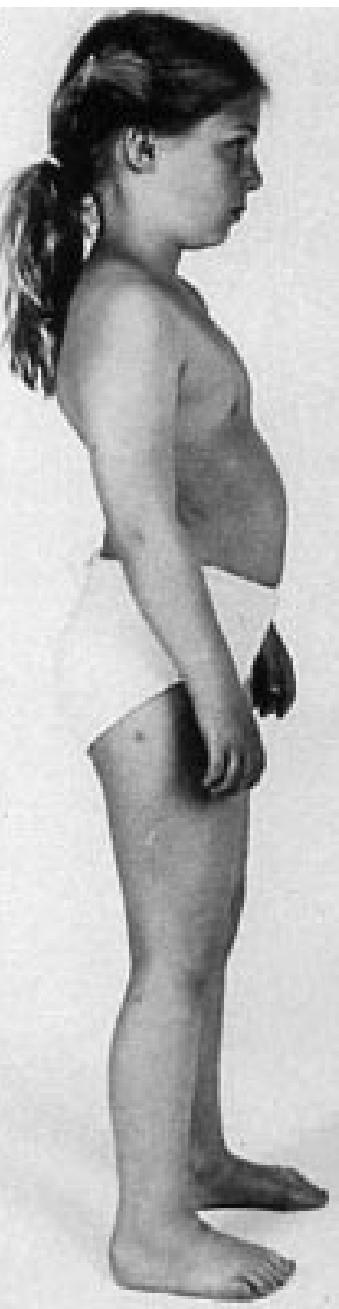

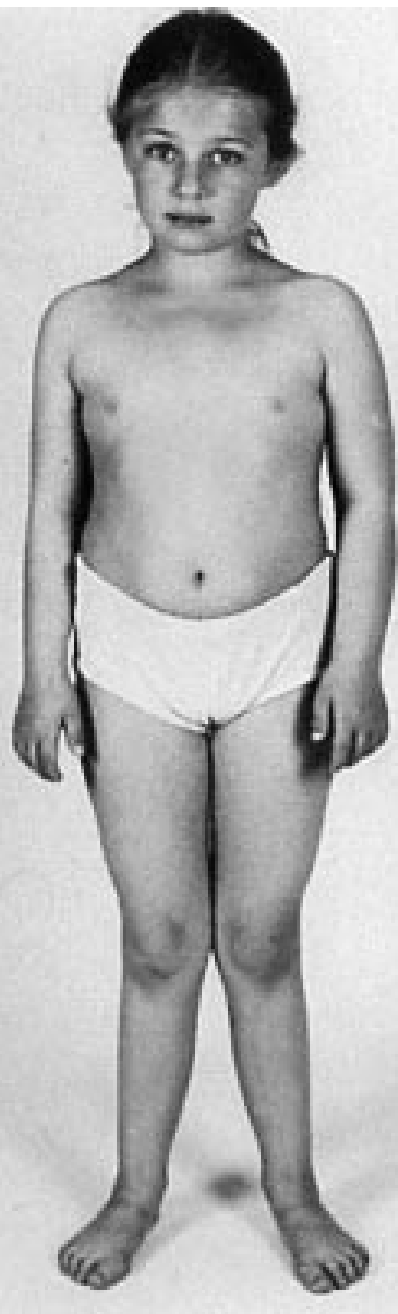

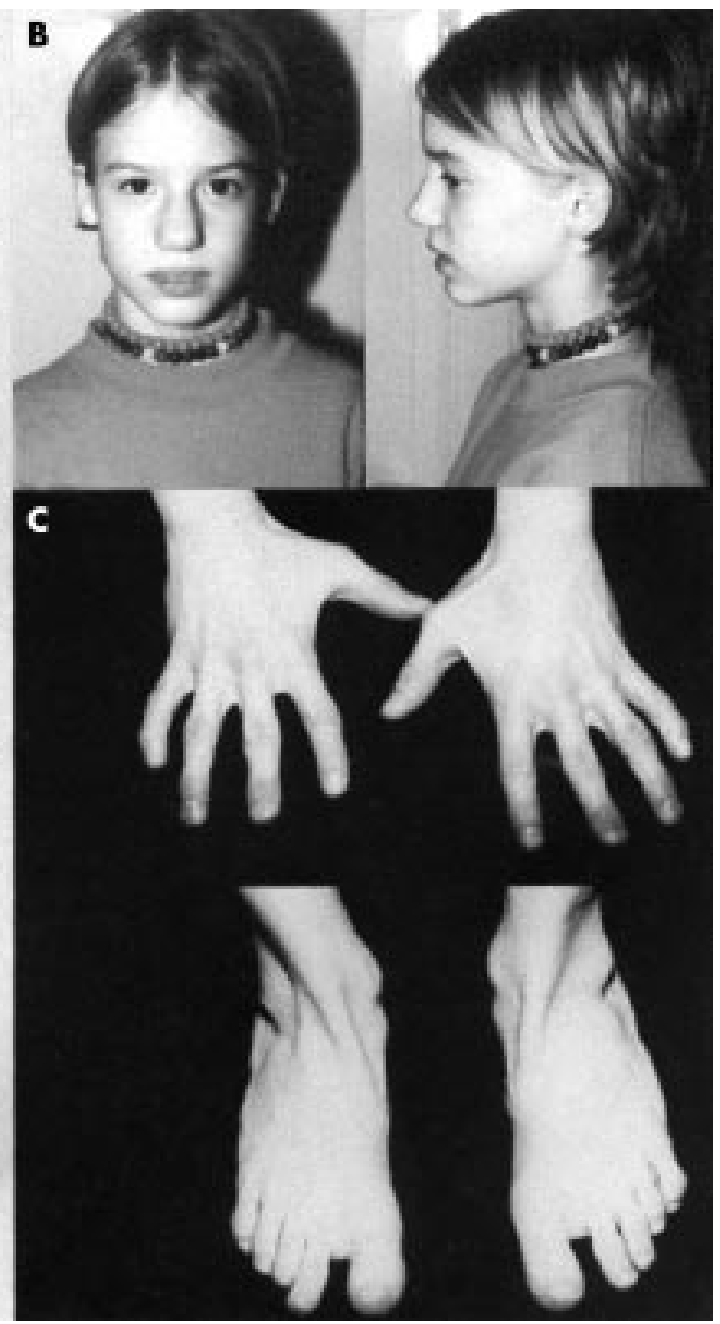

B

Figure 1 (A) Patient 7 at 10 years of age. Her stature was at the 10th centile for age. There are bilateral genua valga and the fingers are moderately short, without hitch-hiker thumb. Her habitus is otherwise unremarkable and not suggestive of a skeletal dysplasia. The ears are not deformed. (B, C) Patient 2 at the age of 13 years. The face (B) is typical of DTD with a long and bulbous nose and distinct from the flat bridged nose seen in other skeletal dysplasias. Note the ears lacking the "cauliflower" deformity typical of diastrophic dysplasia. The hands (C) show slight contractures of the fingers as well as the "bracket" clinodactyly of digits 2 and 5 (ulnar deviation of 2 and radial deviation of 5) which is typical of, although not exclusive to, rMED. These changes, together with hip pain, had suggested a diagnosis of a rheumatoid disorder. There is no hitch-hiker thumb. The feet (C) show slight broadening of the space between the first and second toes but no pes adductus or frank club foot deformity.

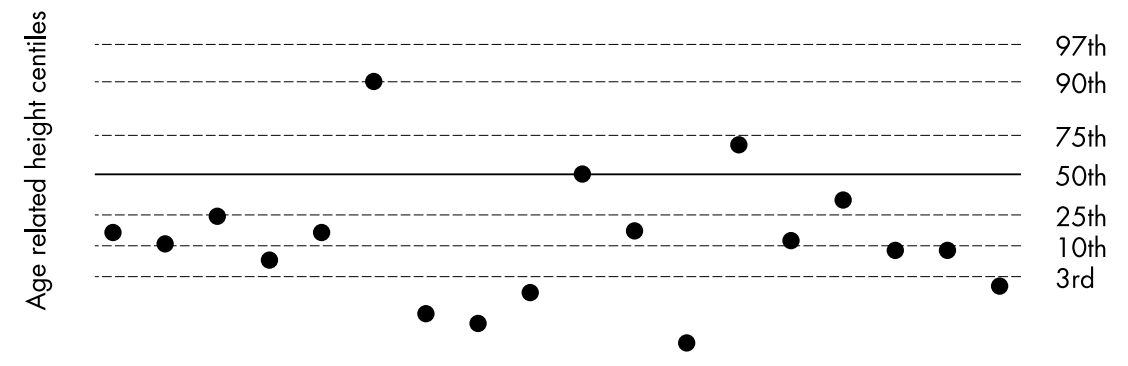

$\begin{array}{llllllllllllllllll}\text { Age (y) } & 9 & 9 & 10 & 10 & 14 & 15 & 20 & 20 & 28 & 28 & 32 & 35 & 36 & 38 & 49 & \mathrm{Ad} & \mathrm{Ad} \\ \mathrm{Ad}\end{array}$

$\begin{array}{llllllllllllllllllll}\text { Patient No } & 10 b & 9 & 10 a & 7 & 2 & 8 & 3 a & 3 b & 1 & 5 b & 5 a & 11 & 12^{*} & 6 b & 6 a & 4 a & 4 b & 4 c\end{array}$

Figure 2 Height of R279W homozygotes shown in relation to age related centiles (adult centiles for patients over 18 years of age).

bones, with carpal bones showing rather advanced ossification as compared to bone age standards, leading to a reduced carpal width in patients around the age of 10 and older. The phalanges tend to be broad and short giving moderate brachydactyly. The phalangeal epiphyses tend to be small, simulating delayed bone development. Their shape was normal in most patients; a single patient had cone shaped epiphyses of the proximal phalanges (fig 3 ).

Pelvis (fig 4)

The most frequent and suggestive finding in the pelvic $x$ rays was abnormal capital femoral epiphyses. In childhood there 

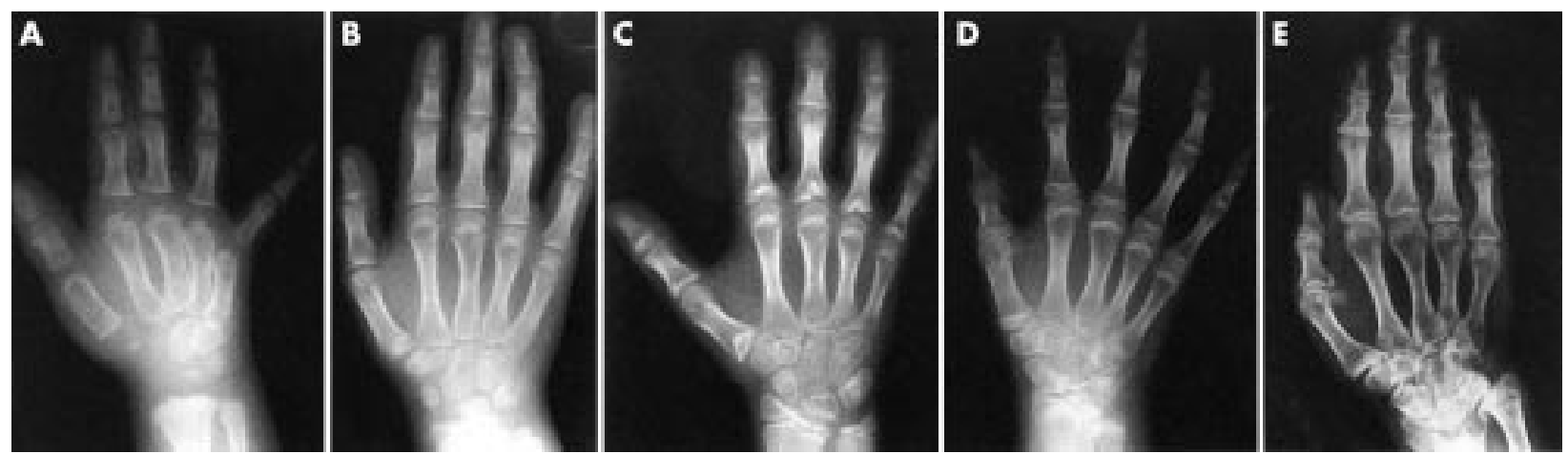

Figure 3 Hand $x$ rays of five different patients of increasing age. (A) Patient 1 (female, skeletal age 3 years 6 months). (B) Patient 8 (male, 8 years, skeletal age 8 years). (C) Patient 7 (female, 9 years, skeletal age 10 years). (D) Patient 9 (female, 13 years, skeletal age 13 years 6 months). (E) Patient $6 \mathrm{~b}$ (female, 37 years). The carpal region shows advanced maturation compared to age standards but is smaller than normal, particularly in the 13 year old and adult hands (D, E). The metacarpals and phalanges are stubby but otherwise not remarkable. The distal metacarpal and phalangeal epiphyses are slightly flattened with regular contours. Cone shaped epiphyses are seen in all proximal phalanges of patient 7 but not in other patients.

may be epiphyseal flattening without frank dysplasia (fig 4A), but in subjects aged 10 or older the epiphyses are thin and crescent shaped. At the same time, the femoral neck tends to become broad and to assume a valgus position (fig $4 \mathrm{~B}$ shows pronounced changes seen in patient 8 at the same age as that of (A)). These changes are rather characteristic of the earlier "Ribbing type" MED. In adult patients, where the thin epiphyses are no longer discernible, the femoral neck appears short.

\section{Knees (fig 5)}

A lateral $x$ ray of the knee was performed in 10 of 18 patients; seven (two-thirds) of them showed a double layered patella. Their age range was between 8 and 28 years. Thus, unlike the double layered sternum seen only in young children with $\mathrm{DTD}_{,}{ }^{24}$ the double layered patella persists into early adulthood. This probably indicates that it is caused not merely by an abnormal ossification pattern but is a true anatomical variant, with two ossific nuclei instead of one. Although we cannot be sure how many rMED patients have a double layered patella (diagnostic sensitivity), specificity of this sign may be high, as all patients with double layered patella referred to us turned out to have DTDST mutations (including R279W homozygosity or other genotypes).

\section{Spine}

Changes in the spine were least marked. Platyspondyly or other structural changes of the vertebral bodies were not observed. Mild lumbar scoliosis was observed occasionally, but this may have been the consequence of lower limb and foot deformity. In most patients, the interpedicular distance in the L1 to L5 segment remained constant or decreased, as usually observed in diastrophic dysplasia.

\section{DISCUSSION}

Following the identification of the DTDST gene, molecular diagnosis of subjects affected by DTD, AO2, or ACG1B has shown that c862t/R279W is the most frequent DTDST mutation, but it was never found in the homozygous state. Homozygosity for R279W was finally observed in a patient who had double layered patella, club foot, and normal stature and had received the diagnosis of multiple epiphyseal dysplasia. ${ }^{20}$ Following the description of this person, several other homozygotes were found in a relatively short time. ${ }^{1321} 22$ In this study, we have delineated the clinical and radiographic phenotype of 18 R279W homozygotes. Subjects with the same pathogenic genotype are rare in the skeletal dysplasias (with the notable exception of achondroplasia). This gave us the opportunity of defining the phenotype and of estimating the degree of clinical variability resulting from other genetic and epigenetic factors.
The results of this study confirm the existence of a recessive form of MED situated at the mild end of the dysplasia spectrum that includes achondrogenesis $1 \mathrm{~B}$, atelosteogenesis 2 , and diastrophic dysplasia. The finding of normal stature and the rarity of abnormal findings at birth (particularly of cleft palate, hitch-hiker thumbs, and cystic ear swelling) clearly distinguishes rMED from diastrophic dysplasia and justifies the descriptive term "multiple epiphyseal dysplasia", as has been applied to several patients in the past. ${ }^{20}$ The diagnosis of rMED is usually made in childhood because of joint complaints and mild hand and foot deformity. Stature is slightly reduced on average, with some patients below the normal mean and others above.

Because of the relatively mild radiographic findings, differential diagnosis of the various MED types is difficult. However, this differential diagnosis is important, as it should form the basis of further molecular diagnostic studies. Unger et al $^{14}$ have delineated radiographic criteria to distinguish COMP associated MED (with hip involvement more pronounced than knee involvement) from collagen 9 associated MED (where the hips are relatively spared) ${ }^{14}$ The hip changes in MED, with broad and flat epiphyses, are quite consistent in our series and easy to distinguish from those in COMP MED, with small and round epiphyseal nuclei. On hand radiographs, rMED patients may be identified by slightly flattened epiphyses sitting on relatively broad metacarpal ends. The extent of brachydactyly is similar to that seen in COMP MED and greater than that in collagen 9 MED. The finding of even mild club foot deformity (present in five of 18 patients in this series) is suggestive of rMED as it is not commonly seen in other MED types. The observation of a double layered patella on lateral radiographic films is strongly suggestive of rMED; unfortunately, the nonsystematic nature of skeletal surveys in our patient series does not allow statements on the sensitivity of this sign or on the time frame in which it can be observed. It is surprising that in spite of the evident changes at the hips, no subject has undergone hip replacement surgery. This is in contrast with the findings in COMP associated MED, where hip surgery is common. The reason for this difference might be the ligamentous laxity and joint instability in COMP MED as opposed to the tendency to joint contractures in rMED.

The stature range of R279W homozygotes was even larger than that of the reference population, with some of them being below the 3rd centile for height and others being tall-normal. While the median height is below the normal mean, most patients fall within the low normal range. This wide stature range is at odds with the expectation of a skeletal dysplasia having a major impact on stature. The reasons for this variability are unclear. Parental height is a major determinant of individual height; unfortunately, this information was 

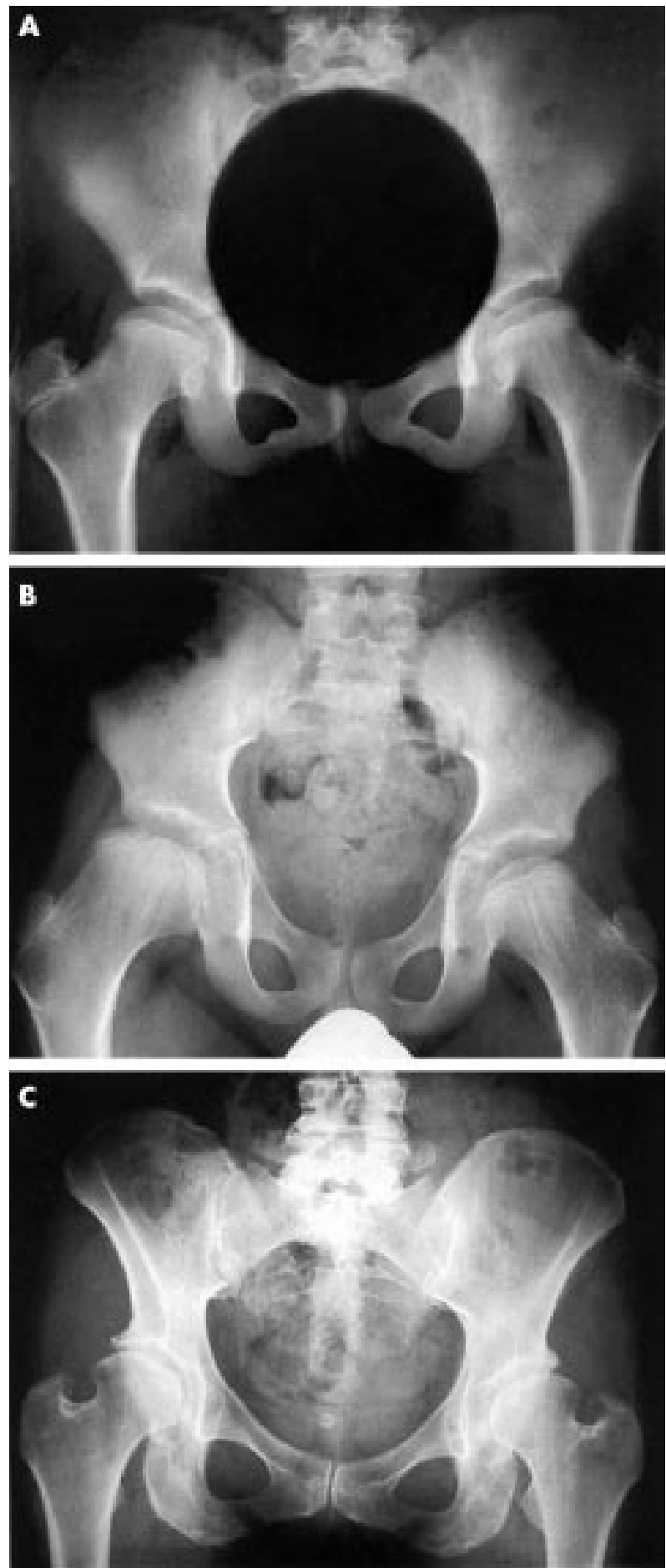

Figure 4 The spectrum of radiographic changes in the pelvis and hips. (A) Patient 7 (female, 9 years). (B) Patient 8 (male, 9 years). (C) Patient $6 \mathrm{~b}$ (female, 37 years). In a 9 year old girl (A), the proximal femoral epiphyses are small and less rounded than usual, but not frankly dysplastic. In a boy of the same age, more pronounced changes are seen: the epiphyses are flattened and the femoral necks are broad and show a valgus deformation. In the adult, the epiphyses are no longer discernible and the whole femoral neck is short.

unavailable to us. Genetic variance in our ethnically heterogeneous group of R279W homozygotes may be greater than in the central European reference population used to calculate the growth curves. The factors determining body height in
rMED should be studied more accurately, as adult stature is an important general outcome criterion in skeletal dysplasias.

Following the description of the first R279W homozygote, we put forward the hypothesis that DTDST mutations are responsible for a subset of cases of idiopathic club foot. ${ }^{2021}$ Indeed, Huber et $a l^{22}$ described four R279W homozygotes in two families who had presented at birth with "apparently isolated" club foot, and in whom later examination had shown rMED. We tested a large sample of families with familial idiopathic talipes equinovarus for the presence of DTDST mutations but did not find any. ${ }^{25}$ Interestingly, though, we found heterozygosity for R279W in two unaffected subjects, probably as a chance finding (see below). In the present series, club foot was seen in six out of 18 subjects, confirming that it is a frequent but not obligatory feature of rMED. It seems prudent to conclude that children with bilateral club foot should be examined for the presence of rMED but that the contribution of DTDST mutations to the pathogenesis of club foot as a whole is probably small.

Unlike all other MED types known so far, this type of MED is inherited as a recessive trait (rMED), which has implications for genetic counselling. Recurrence probability in sibs is $25 \%$. For an affected subject, the probability of transmitting the disorder to offspring is not $50 \%$ but significantly lower. For such a subject, the risk of having a child with a DTD skeletal dysplasia (rMED, DTD, AO2, or ACG1B) may be around 1 in 200 (assuming a carrier frequency of around 1 in 100 for DTDST mutations altogether ${ }^{19}$ ). In spite of the relatively low risk, screening of the partner for the most common DTDST mutations (including R279W) is an option that is frequently used. ${ }^{19}$

It would be of interest to estimate what proportion of MED cases might be ascribed to DTDST associated rMED. The incidence of $\mathrm{rMED}$, and the proportion of MED cases that is accounted for by rMED, cannot be determined with precision by this retrospective case collection. Nonetheless, some indications can be derived from this study and from published reports. Occurrence of $\mathrm{c} 862 \mathrm{t} / \mathrm{R} 279 \mathrm{~W}$ at a $\mathrm{CpG}$ dinucleotide might be consistent with recurrent mutational events. R279W is the only DTDST mutation that has been found fortuitously in the heterozygous state in unaffected subjects (two of 207 samples in one study ${ }^{25}$ ). Homozygosity may thus be more common than hitherto recognised. Since we have observed the rMED phenotype in seven other subjects with DTDST genotypes other than R279W homozygosity, rMED is not exclusively associated with R279W, although this genotype appears to be the most frequent. Altogether, rMED has been proven by mutation analysis in a total of 28 subjects in our laboratory. Czarny-Ratajczak et al $^{13}$ screened a large sample of MED patients and found three instances of COMP mutations, one of COL9A1, none of COL9A2 or COL9A3, and two unrelated $\mathrm{R} 279 \mathrm{~W}$ homozygous probands; more probands in that series had a double layered patella but other DTDST mutations were not tested for. Huber $e a^{22}$ reported four R279W homozygous rMED subjects. Thus, DTDST associated rMED constitutes at present the largest subgroup of molecularly defined MED patients. These observations suggest that rMED may not be uncommon. Given the variability observed in our series, it is conceivable that some patients with rMED might never be diagnosed with a generalised skeletal disorder or might even escape medical attention. The data presented here can help increase recognition of rMED by clinicians.

\section{ACKNOWLEDGEMENTS}

This work was supported by the Swiss National Foundation (grant 31-57272.99) and the National Institutes of Health (grant HD 22657). We are grateful to Outi Makitie and Ravi Savarirayan for sharing information on non-R279W rMED patients, and to Leena Ala-Kokko and Beat Steinmann for constructive discussions and for reviewing the manuscript. 

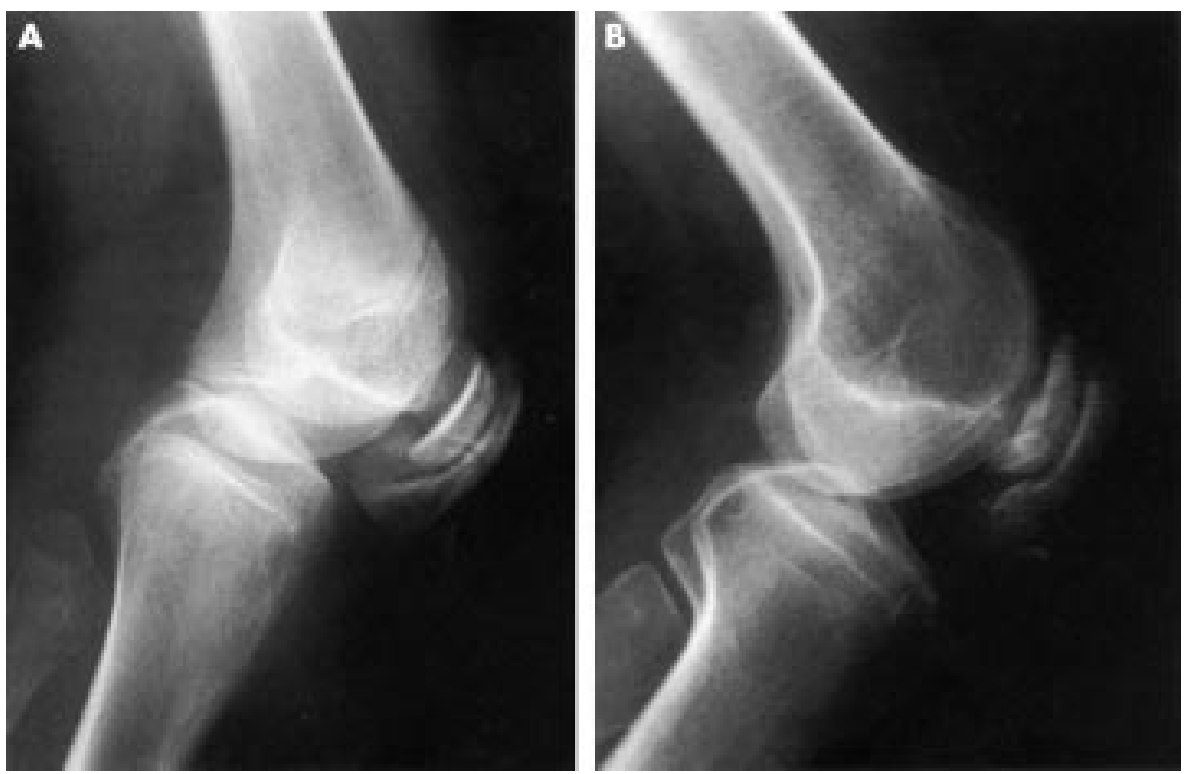

Figure 5 Lateral knee $x$ ray of $(A)$ patient $3 a$ at 20 years and (B) patient $5 b$ at 28 years showing a double layered patella. Persistence of this finding into adulthood indicates a true anatomical variant and is a useful diagnostic clue.

Table 2 Overview on molecularly defined types of multiple epiphyseal dysplasia

\begin{tabular}{lllll}
\hline & MIM number & Gene & Chromosomal mapping & Inheritance \\
\hline EDM1 & $* 600310$ & COMP & $19 p 13.1$ & Dominant \\
EDM2 & 600204 & COL9A2 & $1 p 33-p 32$ & Dominant \\
EDM3 & 600969 & COL9A3 & $20 q 13.3$ & Dominant \\
EDM4 & 226900 & DTDST & $5 \mathrm{q} 32-q 33.1$ & Recessive \\
- & $* 120210$ & COL9A1 & $6 q 13$ & Dominant \\
EDM5 & $* 602109$ & MATN3 & $2 p 24-p 23$ & Dominant \\
\hline
\end{tabular}

...............

\section{Authors' affiliations}

D Ballhausen, L Bonafé, A Superti-Furga, Division of Metabolism and Molecular Paediatrics, University Children's Hospital, Zürich, Switzerland P Terhal, Department of Medical Genetics, Wilhelmina Children's Hospital, Utrecht, The Netherlands

S L Unger, D H Cohn, Ahmanson Department of Pediatrics, Burns and Allen Cedars Sinai Research Institute, and Departments of Human Genetics and Pediatrics, UCLA School of Medicine, Los Angeles, USA

G Bellus, Department of Dermatology, University of Colorado Health Sciences Center, Denver, Colorado, USA

M Classen, Zentralkrankenhaus Links der Weser, Bremen, Germany

B C Hamel, Department of Human Genetics, University Hospital,

Nijmegen, The Netherlands

J Spranger, Greenwood Genetic Center, Greenwood, South Carolina, USA

B Zabel, Kinderklinik, Johannes Gutenberg Universität, Mainz, Germany W G Cole, Division of Orthopaedic Surgery, The Hospital for Sick Children, Toronto, Ontario, Canada

J T Hecht, Departments of Pediatrics and Medical Genetics, University of Texas Medical School, Houston, Texas, USA

Correspondence to: Professor A Superti-Furga, Division of Molecular Paediatrics, Centre Hospitalier Universitaire Vaudois, $\mathrm{CH}-1011$

Lausanne, Switzerland; asuperti@chuv.unil.ch

\section{REFERENCES}

1 Ribbing S. Studien über hereditäre, multiple Epiphysenstörungen. Acta Radiol 1937;suppl 34: 1-107.

2 Fairbank HAT. Dysplasia epiphysealis multiplex. Proc $R$ Soc Med Lond 1946:39:315-17

3 International Working Group on Constitutional Diseases of Bone. International nomenclature and classification of the

osteochondrodysplasias (1997). Am J Med Genet 1998;79:376-82.

4 Hecht JT, Nelson LD, Crowder E, Wang Y, Elder FFB, Harrison WR

Francomano CA, Prange CK, Lennon GG, Deere M, Lawler J. Mutations in exon 17B of cartilage oligomeric matrix protein (COMP) cause pseudoachondroplasia. Nat Genet 1995;10:325-9.

5 Briggs MD, Hoffman SMG, King LM, Olsen AS, Mohrenweiser H, Leroy JG, Mortier GR, Rimoin DL, Lachman RS, Gaines ES, Cekleniak JA Knowlton RG, Cohn DH. Pseudoachondroplasia and multiple epiphyseal dysplasia due to mutations in the cartilage oligomeric matrix protein gene. Nat Genet 1995;10:330-6.

6 Holden P, Meadows RS, Chapman KL, Grant ME, Kadler KE, Briggs M. Cartilage oligomeric matrix protein interacts with type IX collagen, and disruptions to these interactions identify a pathogenic mechanism in a bone dysplasia family. J Biol Chem 2001;276:6046-55.

7 Thur J, Rosenberg K, Nitsche DP, Pihlajamaa T, Ala-Kokko L, Heinegard D. Paulsson M, Maurer P. Mutations in cartilage oligomeric matrix protein (COMP) causing pseudoachondroplasia and multiple epiphyseal dysplasia affect binding of calcium and collagen I, II and IX. J Biol Chem 2001;276:6083-92

8 Holden P, Canty EG, Mortier GR, Zabel B, Spranger J, Carr A, Grant $M E$, Loughlin JA, Briggs MD. Identification of novel pro-alpha2(IX) collagen gene mutations in two families with distinctive oligo-epiphyseal forms of multiple epiphyseal dysplasia. Am J Hum Genet 1999;65:31-8.

9 Muragaki Y, Mariman EC, van Beersum SE, Perala M, van Mourik JB, Warman ML, Olsen BR, Hamel BC. A mutation in the gene encoding the alpha 2 chain of the fibril- associated collagen IX, COL9A2, causes multiple epiphyseal dysplasia (EDM2). Nat Genet 1996;12:103-5.

10 Warman ML, McCarthy MT, Perala M, Vuorio E, Knoll JH, McDaniels $\mathrm{CN}$, Mayne R, Beier DR, Olsen BR. The genes encoding alpha 2 (IX) collagen (COL9A2) map to human chromosome 1p32.3-p33 and mouse chromosome 4. Genomics 1994;23:158-62.

11 Paassilta P, Lohiniva J, Annunen S, Bonaventure J, Le Merrer M, Pai L, Ala-Kokko L. COL9A3: A third locus for multiple epiphyseal dysplasia (published erratum appears in Am J Hum Genet 1999;65:1214). Am J Hum Genet 1999;64:1036-44.

12 Tiller GE, Warman ML, Gong Y, Knoll JH, Mayne R, Brewton RG. Physical and linkage mapping of the gene for the alpha3 chain of type IX collagen, COL9A3, to human chromosome 20q13.3. Cytogenet Cell Genet 1998;81:205-7.

13 Czarny-Ratajczak M, Lohiniva J, Rogala P, Kozlowski K, Perala M, Carter L, Spector TD, Kolodziej L, Seppanen U, Glazar R, Krolewski J, Latos-Bielenska A, Ala-Kokko L. A mutation in COL9Al causes multiple epiphyseal dysplasia: further evidence for locus heterogeneity. Am J Hum Genet 2001;69:669-80. 
14 Unger SL, Briggs MD, Holden P, Zabel B, Ala-Kokko L, Paassilta P, Lohiniva J, Rimoin DL, Lachman RS, Cohn DH. Multiple epiphyseal dysplasia: radiographic abnormalities correlated with genotype. Pediatr Radiol 2001;31:10-18.

15 Chapman KL, Mortier GR, Chapman K, Loughlin J, Grant ME, Briggs $M D$. Mutations in the region encoding the von Willebrand factor $A$ domain of matrilin-3 are associated with multiple epiphyseal dysplasia. Nat Genet $2001 ; 28: 393-6$.

16 Hastbacka J, de la Chapelle A, Mahtani MM, Clines G, Reeve-Daly MP, Daly M, Hamilton BA, Kusumi K, Trivedi B, Weaver A, Coloma A, Lovett $M$, Buckler A, Kaitila I, Lander ES. The diastrophic dysplasia gene encodes a novel sulfate transporter: positional cloning by fine-structure linkage disequilibrium mapping. Cell 1994;78:1073-87.

17 Hastbacka J, Superti-Furga A, Wilcox WR, Rimoin DL, Cohn DH, Lander ES. Atelosteogenesis type II is caused by mutations in the diastrophic dysplasia sulfate-transporter gene (DTDST): evidence for a phenotypic series involving three chondrodysplasias. Am J Hum Gene 1996;58:255-62

18 Superti-Furga A, Hastbacka J, Wilcox WR, Cohn DH, van der Harten HJ, Rossi A, Blau N, Rimoin DL, Steinmann B, Lander ES, Gitzelmann R. Achondrogenesis type IB is caused by mutations in the diastrophic dysplasia sulphate transporter gene. Nat Genet 1996;12:100-2.

19 Rossi A, Superti-Furga A. Mutations in the diastrophic dysplasia sulfate transporter (DTDST) gene (SLC26A2): 22 novel mutations, mutation review, associated skeletal phenotypes, and diagnostic relevance (erratum appears in Hum Mutat 2001;18:82). Hum Mutat 2001;17:159-71.

20 Superti-Furga A, Neumann L, Riebel T, Eich G, Steinmann B, Spranger J, Kunze J. Recessively inherited multiple epiphyseal dysplasia with normal stature, club foot, and double layered patella caused by a DTDST mutation. J Med Genet 1999;36:621-4.

21 Superti-Furga A, Sobetzko D, Hecht JT, Unger SL, Cole WG, Hamel BC, Bellus G, Classen M, Le Merrer M, Zabel B, Langer LO, Spranger J, Cohn DH. Recessive multiple epiphyseal dysplasia (rMED; MIM 226900): phenotype delineation in twelve individuals homozygous for DTDST mutation R279W (abstract). Am J Hum Genet 2000;67:379.

22 Huber C, Odent S, Rumeur S, Padovani P, Penet C, Cormier-Daire V, Munnich A, Le Merrer M. Sulphate transporter gene mutations in apparently isolated club foot. J Med Genet 2001;38:191-3.

23 Deere M, Blanton SH, Scott Cl, Langer LO, Pauli RM, Hecht JT. Genetic heterogeneity in multiple epiphyseal dysplasia. Am J Hum Genet 1995; 56:698-704

24 Currarino G. Double-layered manubrium sterni in young children with diastrophic dysplasia. Pediatr Radiol 2000;30:404-9.

25 Bonafé L, Blanton SH, Scott A, Broussard S, Wise CA, Superti-Furga A, Hecht JT. DTDST mutations are not a frequent cause of idiopathic talipes equinovarus (club foot). J Med Genet 2002;39:e20.

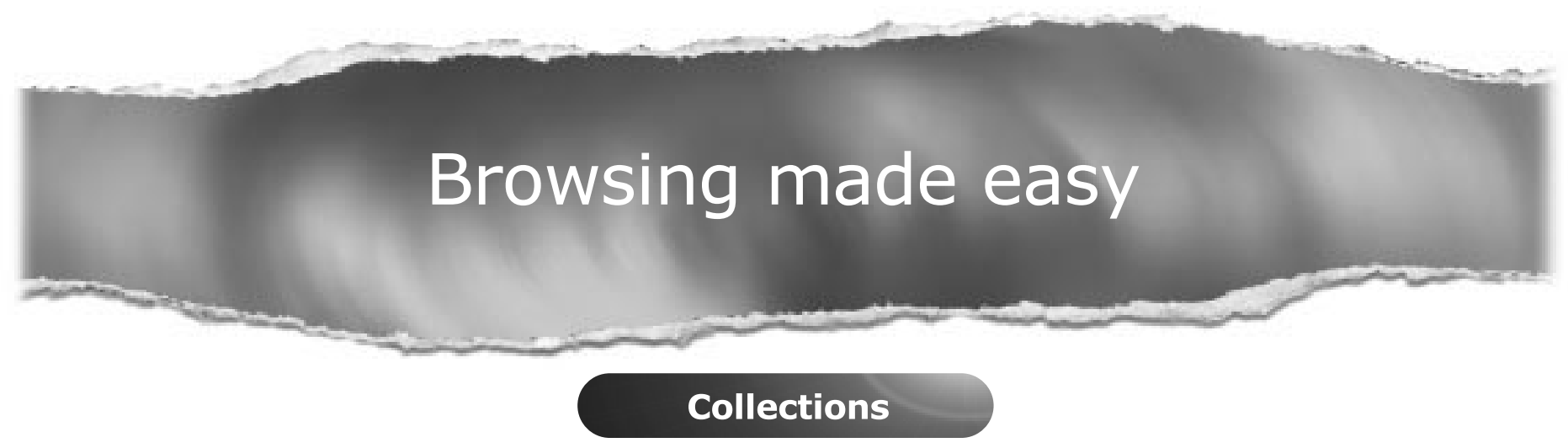

With a single click Collections allows you to find all articles that have been published in your chosen subject. Select from over 200 clinical and non-clinical topic collections and/or cross search other specialist journals, the BMJ and Cochrane Reviews

www.jmedgenet.com 